\title{
Integração à Vida Acadêmica entre Alunos de Curso de Educação Geral
}

\section{General Education Students Engagement and Belonging in Academic Life}

\section{Integración a la Vida Académica entre Alunos de Curso de Educación General}

\author{
Soely A J Polydoro \\ Docente do Departamento de Psicologia Educacional da Faculdade de Educação da Unicamp \\ Ana Maria Alves Carneiro \\ Pesquisadora do Núcleo de Estudos de Políticas Públicas (NEPP) da Unicamp \\ *Agência de fomento: $\mathrm{CNPq}$
}

\begin{abstract}
Resumo
Este artigo explora o processo de integração ao ensino superior, seus domínios e impactos no desempenho acadêmico de 168 estudantes do Programa de Formação Interdisciplinar Superior (ProFIS), turmas de 2012 e 2013, da Universidade Estadual de Campinas (Unicamp). Os dados foram obtidos por meio da utilização de uma escala sobre a vida acadêmica com 34 itens distribuídos nas dimensões: ambiente universitário, compromisso com o curso, habilidade do estudante, envolvimento em atividades não obrigatórias e condições para o estudo. Os resultados de integração foram discutidos em relação ao momento do curso (final do primeiro e do segundo ano), situação acadêmica (conclusão ou ativo no curso) e desempenho acadêmico (coeficiente de rendimento). Dado o papel da integração à vida universitária para o sucesso do estudante, o artigo ressalta o desafio posto à gestão institucional em sua promoção.
\end{abstract}

Palavras-chave: desempenho acadêmico; educação universitária; coeficiente de rendimento.

Abstract

This article explores the process of engagement and belonging in academic life, its domains and impacts on academic performance of 168 students of Interdisciplinary Higher Education Program (PROFIS) from the University of Campinas (Unicamp) - 2012 and 2013 classes. The data were obtained by the use of a scale of academic life, with 34 items, distributed in the dimensions: university environment, commitment to the course, student skills, involvement in non-mandatory activities and conditions for the study. The results about engagement and belonging in academic life were discussed in relation to the time of the course (end of the first and second year), academic status (active or graduated) and academic performance (coefficient of performance). Given the role of engagement into university life to student success, the article highlights the challenge faced by the institutions for manage the program.

Keywords: academic performance; higher education; coefficient of performance.

Resumen

Este artículo estudia el proceso de integración a la enseñanza superior, sus campos e impactos en el desempeño académico de 168 estudiantes del Programa de Formação Interdisciplinar Superior (ProFIS), clases de 2012 a 2013, de la Universidade Estadual de Campinas (UNICAMP). Los datos fueron obtenidos através de la utilización de una escala sobre la vida académica con 34 ítems distribuidos en las dimensiones: entorno universitário, compromiso con el curso, habilidad del estudiante, participación en actividades no obligatorias y condiciones para el estudio. Los resultados de integración fueron discutidos en relación al momento del curso (final del primero y del segundo año), situación académica (conclusión o activo en el curso) y desempeño académico (coeficiente de rendimento). Teniendo en cuenta el papel de la integración a la vida universitária para el éxito del estudiante, el artículo destaca el desafio puesto a la gestión institucional en su promoción.

Palabras-clave: desempeño académico; educación universitária; coeficiente de rendimiento. 
Este texto tem por objetivo apresentar uma análise da percepção dos estudantes, do Programa de Formação Interdisciplinar Superior (ProFIS) da Universidade Estadual de Campinas (Unicamp), sobre sua vivência universitária. O ProFIS é um curso sequencial de complementação de estudos que objetiva possibilitar uma formação geral, de caráter multidisciplinar, que proporcione aquisição de conhecimentos em Ciências Humanas, Ciências da Natureza e Arte, visando abordagens integradas sobre o conhecimento, suas relações com o mundo, com o ambiente de trabalho, bem como a compreensão de si mesmos como indivíduos e cidadãos de uma sociedade diversificada, globalizada e em constante mudança (Pró-reitoria de Graduação, 2010).

Além da proposta de educação geral, o programa objetiva ainda a inclusão de estudantes oriundos exclusivamente de escolas públicas do município de Campinas. A seleção é realizada de forma diferenciada por meio de um sistema que combina a distribuição de vagas para as escolas públicas da cidade de Campinas e a nota do Exame Nacional do Ensino Médio (ENEM). São oferecidas 120 vagas anuais para os alunos melhores classificados pelo ENEM - de cada uma das 96 escolas públicas de Campinas que oferecem o ensino médio, são aceitos no máximo dois matriculados por escola.

O programa é estruturado em dois ciclos: $\mathrm{O}$ primeiro é o curso de educação geral com duração mínima de 2 anos. Os estudantes do ProFIS vão, ao longo do curso, conhecendo os cursos e carreiras a partir do contato com as diversas disciplinas, institutos e faculdades, experiências universitárias e, por fim, cursam a disciplina "Profissões" como forma de Orientação Profissional para uma escolha mais segura e madura sobre carreira. Os alunos também têm a oportunidade de engajamento em pesquisa sob orientação de um professor da Unicamp de qualquer área do conhecimento, seja inserido no cotidiano do processo criativo e/ou no processo de pesquisa em um laboratório ou de atividade de arte, desenvolvendo um projeto de iniciação científica. Após a conclusão do ProFIS, os estudantes são matriculados nas vagas reservadas em praticamente todos os cursos da Unicamp. A seleção acontece a partir do ranqueamento dos alunos segundo o coeficiente de rendimento das disciplinas obrigatórias cursadas.

Diante dessa proposta institucional inovadora, torna-se ainda mais relevante compreender como os estudantes percebem sua vivência acadêmica. Os estudantes ingressam em um contexto muito mais complexo em relação à trajetória escolar anterior, mas também novo para a universidade e toda sua comunidade.

A integração à vida acadêmica não é um processo padronizado, “tamanho único". Também não é algo que acontece somente no momento do ingresso, se renova a cada momento do curso (Tinto, 1997), na relação recíproca entre estudante (percepções, escolhas, objetivos e ações) e ambiente de formação (normas, expectativas e oportunidades) (Dey \& Hurtado, 1995). Dado constante é que, para integrar-se, o estudante ingressante necessitará enfrentar desafios propostos de natureza acadêmica, social, pessoal, de carreira e institucional (Almeida \& Soares, 2003; Almeida et al., 2004). Desafios que, muitas vezes, associam-se à necessidade de gestão dos recursos financeiros, organização e administração do espaço de moradia, transporte, cuidados com a alimentação, saúde e lazer. Ou ainda, conciliar as demandas advindas das atividades externas à instituição, como atividade de trabalho e responsabilidades familiares, com aquelas relacionadas ao curso de graduação. Todos esses compromissos exigem do estudante um domínio maior da gestão do tempo e do processo de autorregulação do estudo em direção aos objetivos pessoais. E nessa nova vivência acadêmica, a integração tem sido um dos processos destacados para o sucesso do estudante (Almeida \& Soares, 2003; Santos, Polydoro, Teixeira, \& Bardagi, 2010).

Nesse sentido, com o propósito em colaborar com a avaliação do Programa e o desenvolvimento da área de integração ao ensino superior, o presente estudo tem como objetivos: comparar os escores de integração à vida acadêmica dos estudantes das turmas de 2012 e 2013 do ProFIS ao final do primeiro ano de curso; comparar os escores de integração à vida acadêmica dos estudantes 
da turma de 2012 do ProFIS ao final do primeiro e do segundo ano de curso; analisar a relação entre os escores de integração à vida acadêmica e situação acadêmica e o coeficiente de rendimento dos estudantes em disciplinas obrigatórias.

\section{Método}

\section{Participantes}

Os dados que seguem referem-se à percepção de 168 estudantes do ProFIS sobre sua integração à vida acadêmica, sendo que 89 ingressaram em 2012 (74\% do total de matriculados naquele ano) e 79 em 2013 (66\% do total). Para a turma de 2012, além da coleta ao final do primeiro ano, realizou-se outra coleta ao final do segundo ano, da qual participaram 63 alunos (53\% do total).

Dentre os participantes, $110(59,52 \%)$ continuavam ativos no ProFIS, 62 (36,90\%) tinham concluído o curso e seis evadido (3,57\%) ao final de 2013.Quanto ao desempenho acadêmico em disciplinas obrigatórias ao final do primeiro ano do curso, os estudantes da turma de 2012 tinham coeficiente médio de $0,67(D P=0,15) \mathrm{e}$ os da turma de 2013 apresentavam coeficiente médio de $0,62(D P=0,18)$.

\section{Instrumento}

Para obter a percepção dos estudantes sobre sua vivência universitária, foi utilizada a Escala sobre a Vida Acadêmica (EAVA) (Vendramini et al., 2004). Trata-se de um instrumento com 34 itens distribuídos em cinco dimensões, a saber: 1) ambiente universitário (oito itens, avalia a percepção do estudante em relação ao contexto físico, social, acadêmico e organizacional da instituição), 2) compromisso com o curso (sete itens sobre o grau de certeza do estudante quanto a sua escolha de curso e sobre a percepção de segurança quanto à formação propiciada pelo curso), 3) habilidade do estudante (10 itens a respeito da percepção do estudante sobre suas habilidades de aprendizagem e background decorrente da escolaridade prévia), 4) envolvimento em atividades não obrigatórias (cinco itens que avaliam a integração do estudante com experiência de caráter acadêmico, científico, social, cultural ou esportivo propiciadas pela instituição, mas que não são exigências do curso) e 5) condições para o estudo (quatro itens que incluem as condições físicas, disponibilidade de tempo e de investimento nas atividades acadêmicas).

Os estudantes respondem em uma escala de resposta Likert de cinco pontos em contínuo entre discordo totalmente e concordo totalmente. Do total de itens, 16 tem sentido negativo e tiveram sua pontuação invertida para o cálculo das respostas. Para obtenção dos resultados, os pontos foram somados e divididos pelo número de itens de cada dimensão ou do total; quanto maior o valor, maior o nível de integração do estudante à vida acadêmica. Para o estudo atual, só foram incluídas as respostas dos estudantes que responderam à totalidade dos itens.

A confiabilidade da escala foi analisada por meio do coeficiente alfa de Cronbach. Observouse alta consistência interna para a escala total na primeira $(\alpha=0,84)$ e segunda $(\alpha=0,87)$ avaliação. Ressalta-se, no entanto, que a dimensão condições para o estudo teve baixo coeficiente de consistência interna ( $\alpha=0,53$ e $\alpha=0,38$ na primeira e segunda avaliação, respectivamente), muito provavelmente devido ao reduzido número de itens no domínio.

\section{Procedimento}

A escala foi aplicada de forma coletiva na segunda e terceira turma do ProFIS, anos de ingresso de 2012 e 2013, por ocasião da aplicação do questionário de acompanhamento em meados de novembro de 2012 e de 2013, respectivamente. Os dados analisados são provenientes do projeto de Avaliação Continuada do ProFIS, que tem acompanhado a implementação do programa, seus resultados e impactos desde seu início em 2011 (Andrade et al., 2013; Andrade, Gomes, Knobel, \& Carneiro, 2012; Carneiro, Andrade, \& Gonçalves 2012; Carneiro, Andrade, \& Telles, 2012; Carneiro, Camelo, Andrade, \& Telles 2015). 


\section{Resultados}

A exposição dos resultados está organizada em cinco itens, a saber: 1) Análise das turmas de 2012 e 2013 ao final do primeiro ano do curso; 2) Análise comparativa entre as avaliações ao final do primeiro e ao final do segundo ano da turma de 2012; 3) Análise comparativa entre os escores de integração e a situação acadêmica atual dos estudantes no ProFIS; 4) Análise de correlação entre os escores de integração e coeficiente de rendimento nas disciplinas obrigatórias do curso; e 5) Síntese.

\section{Análise das Turmas de 2012 e 2013 ao final do primeiro ano do curso}

Os dados obtidos ao final do primeiro ano das turmas 2012 e 2013 referentes às estatísticas descritivas em cada dimensão, e no total, são apresentados na Tabela 1.

Considerando que as médias poderiam variar de 1 a 5, observou-se que os estudantes apresentaram integração à vida acadêmica ligeiramente superior ao ponto médio da escala $(3,53)$. As dimensões: ambiente universitário e compromisso com o curso foram as que tiveram escore médio mais elevado (4,04 e 4,11, respectivamente). Nas demais dimensões foram obtidas médias inferiores ao escore da escala total, sendo 3,26 em habilidades do estudante, 3,10 em atividades não obrigatórias e 2,74 em condições de estudo.

Ao analisar o conteúdo dos itens que compõem cada uma dessas dimensões, percebeu-se que as dificuldades dos estudantes, ao final do primeiro ano do curso, referem-se principalmente à participação em atividades não obrigatórias de natureza acadêmica (média 2,86 [DP =1,03] no item 6 e 2,80 [ $D P=1,07]$ no item 33); conhecimentos prévios para aprendizagem e desempenho no curso (média 2,00 $[D P=1,13]$ no item 8 e 2,68 [DP = $1,27]$ no item 14); sonolência nas aulas $(2,65$ [DP $=0,90]$ no item 18); e condições de transporte (média 2,51 [DP = 1,22] no item 28).

Para facilitar o entendimento, as análises descritivas obtidas ao final do primeiro ano de cada turma serão apresentadas separadamente, assim como as comparações do escore total e das cinco dimensões entre as turmas de 2012 e 2013 (Tabela 2). Para o estudo da comparação foi utilizado o teste de Mann-Whitney devido à ausência de distribuição normal das variáveis, detectada por meio da aplicação do teste de normalidade de Shapiro-Wilk.

Considerando o nível de significância de $5 \%$, verificou-se uma diferença significativa entre as duas turmas nas dimensões ambiente universitário e compromisso com o curso (médias maiores na turma de 2012), e na dimensão envolvimento em atividades não obrigatórias (média maior na turma de 2013). Percebe-se, portanto, que apesar da média total de integração à vida acadêmica das duas turmas não se diferenciarem, a turma de 2012 encontrava-se significativamente mais integrada nas dimensões ambiente universitário e compromisso com o curso que os estudantes de 2013. E a turma de 2013 estava significantemente mais integrada que a turma

Tabela 1

Análise descritiva dos escores de integração à vida acadêmica ao final do primeiro ano - turmas 2012 e 2013 (N=128)

\begin{tabular}{|c|c|c|c|c|c|c|c|}
\hline Dimensões & Média & D.P. & Mín. & Q1 & Mediana & Q3 & Máx. \\
\hline Ambiente Universitário (Ambiente) & 4,04 & 0,46 & 2,63 & 3,75 & 4 & 4,38 & 5 \\
\hline Compromisso com o Curso (ComprCurs) & 4,11 & 0,65 & 1,57 & 3,57 & 4,14 & 4,64 & 5 \\
\hline Habilidade do Estudante (HabEstud) & 3,26 & 0,54 & 1,7 & 2,9 & 3,3 & 3,6 & 4,6 \\
\hline Envolvimento em Atividades Não obrigatórias (NaoObriga) & 3,1 & 0,75 & 1,2 & 2,7 & 3 & 3,4 & 5 \\
\hline Condições para estudo (Condições) & 2,74 & 0,7 & 1 & 2,25 & 2,75 & 3,25 & 4,5 \\
\hline Total & 3,53 & 0,38 & 2,32 & 3,32 & 3,53 & 3,74 & 4,65 \\
\hline
\end{tabular}


Tabela 2

Comparação dos escores de integração à vida acadêmica ao final do primeiro ano entre as turmas de 2012 e 2013

\begin{tabular}{|c|c|c|c|c|c|c|c|c|}
\hline Coorte & Variável & $\mathrm{n}$ & Média & D.P. & Mín. & Mediana & Máx. & Valor- $p^{*}$ \\
\hline \multirow{6}{*}{2012} & Ambiente & 65 & 4,13 & 0,42 & 3,5 & 4,13 & 5 & 0,037 \\
\hline & ComprCurs & 65 & 4,29 & 0,51 & 3,29 & 4,43 & 5 & 0,006 \\
\hline & HabEstud & 65 & 3,3 & 0,46 & 2,1 & 3,3 & 4,4 & 0,326 \\
\hline & NaoObriga & 65 & 2,94 & 0,57 & 1,6 & 3 & 4,4 & 0,012 \\
\hline & Condições & 65 & 2,78 & 0,67 & 1,25 & 2,75 & 4 & 0,379 \\
\hline & EAVA_total & 65 & 3,59 & 0,29 & 2,97 & 3,56 & 4,26 & 0,119 \\
\hline \multirow{6}{*}{2013} & Ambiente & 63 & 3,93 & 0,49 & 2,63 & 3,88 & 5 & \\
\hline & ComprCurs & 63 & 3,93 & 0,73 & 1,57 & 4 & 5 & \\
\hline & HabEstud & 63 & 3,22 & 0,61 & 1,7 & 3,2 & 4,6 & \\
\hline & NaoObriga & 63 & 3,27 & 0,88 & 1,2 & 3,2 & 5 & \\
\hline & Condições & 63 & 2,69 & 0,73 & 1 & 2,75 & 4,5 & \\
\hline & EAVA_total & 63 & 3,48 & 0,44 & 2,32 & 3,5 & 4,65 & \\
\hline
\end{tabular}

Nota: * Valor-p referente ao teste de Mann-Whitney para comparação das variáveis entre 2 grupos.

de 2012 quanto ao envolvimento em atividades não obrigatórias.

\section{Análise comparativa entre as avaliações ao final do primeiro e segundo ano da turma de 2012}

A Tabela 3, a seguir, apresenta a comparação dos escores da integração acadêmica e suas dimensões na turma de 2012 entre as avaliações realizadas ao final do primeiro e segundo ano do curso. Foi utilizado o teste de Wilcoxon para comparar as amostras relacionadas, devido à ausência de distribuição normal dos escores.

Pelos resultados, verificou-se diferença significativa entre as avaliações nas dimensões habilidades do estudante, envolvimento em atividades não obrigatórias e a média total, demonstrando aumento dos escores entre as coletas de 2012 e 2013.

Apesar do significativo aumento da integração acadêmica dos estudantes, alguns itens permanecem como dificuldades para o estudante ao final do segundo ano de ingresso: falta de conhecimentos prévios para acompanhar o curso (média 1,82 $[D P=1,00]$ no item 8 e média 2,72
$[D P=1,21]$ no item 14) e problema com transporte para a universidade (média 2,21 [DP $=1,21]$ no item 28).

Além disso, a percepção dos estudantes quanto à sua integração nas dimensões ambiente universitário e compromisso com o curso não se alteraram ao longo do período investigado, provavelmente porque já apresentavam escores mais altos na primeira coleta. No entanto, as condições de estudo se mantiveram como os aspectos de menor integração entre os participantes, mesmo após um ano do curso.

\section{Análise comparativa entre os escores de integração e a situação acadêmica dos estudantes no ProFIS}

A Tabela 4 apresenta as comparações dos escores de integração acadêmica e a situação acadêmica da turma de 2012 ao final de 2013, ou seja, após dois anos do ingresso dos estudantes e quando poderiam estar concluindo o curso se estivessem em fase. Dado o reduzido número de evadidos $(n=2)^{1}$, foram comparados os estudantes que concluíram e os que ainda estavam ativos no

\footnotetext{
${ }^{1}$ A seção do questionário que continha a EAVA fazia parte do fluxo do questionário apenas dos alunos ativos no momento de resposta.
} 
Tabela 3

Comparação dos escores de integração à vida acadêmica ao final do primeiro (A) e segundo (B) anos da turma de 2012 ( $n=41)$

\begin{tabular}{|c|c|c|c|c|c|c|}
\hline Variável & Média & D.P. & Mín. & Mediana & máx. & Valor- $p$ \\
\hline AmbienteA & 4,11 & 0,41 & 3,5 & 4 & 5 & 0,501 \\
\hline AmbienteB & 4,16 & 0,47 & 3,25 & 4,13 & 5 & \\
\hline DifAmbiente & 0,05 & 0,44 & $-1,25$ & 0,13 & 1,13 & \\
\hline ComprCursA & 4,28 & 0,52 & 3,29 & 4,43 & 5 & 0,494 \\
\hline ComprCursB & 4,32 & 0,56 & 2,86 & 4,29 & 5 & \\
\hline DifComprCurs & 0,04 & 0,53 & $-1,29$ & 0 & 1,43 & \\
\hline HabEstudA & 3,24 & 0,48 & 2,1 & 3,3 & 4,4 & 0,004 \\
\hline HabEstudB & 3,45 & 0,55 & 2,3 & 3,4 & 4,9 & \\
\hline DifHabEstud & 0,2 & 0,39 & 0,4 & 0,1 & 1,2 & \\
\hline NaoObrigaA & 3 & 0,63 & 1,6 & 3 & 4,4 & $\mathbf{0 , 0 1 7}$ \\
\hline NaoObrigaB & 3,31 & 0,63 & 2 & 3,2 & 5 & \\
\hline DifNaoObriga & 0,31 & 0,8 & -2 & 0,2 & 2 & \\
\hline CondiçõesA & 2,77 & 0,67 & 1,25 & 2,75 & 4 & 0,493 \\
\hline CondiçõesB & 2,88 & 0,56 & 1,75 & 3 & 4 & \\
\hline DifCondições & 0,1 & 0,74 & $-1,25$ & $-0,25$ & 1,75 & \\
\hline EAVA_totalA & 3,57 & 0,3 & 2,97 & 3,56 & 4,26 & 0,012 \\
\hline EAVA_totalB & 3,71 & 0,38 & 3 & 3,68 & 4,79 & \\
\hline DifEAVA_total & 0,14 & 0,33 & $-0,59$ & 0,09 & 0,94 & \\
\hline
\end{tabular}

Nota: * Valor-p referente ao teste de Wilcoxon para amostras relacionadas.

curso. Para essa análise foi utilizado o teste de Mann-Whitney, devido à ausência de distribuição Normal das variáveis.

Pelos resultados, verificou-se diferença significativa entre os grupos "ativos" e aqueles que concluíram o curso no tempo previsto de integralização nas dimensões envolvimento em atividades não obrigatórias medidas ao final do primeiro ano do curso, habilidades do estudante e integração acadêmica, ambas foram medidas no final do segundo ano do curso. A maior média de integração às atividades não obrigatórias foi observada no grupo dos estudantes ativos e a maior média em habilidades do estudante e medida global de integração foi entre os concluintes/ formandos. Se considerarmos a medida limite de significância, podemos inferir que o escore em habilidades do estudante, ao final do primeiro ano, $(p=0,05)$ também foi diferente para os dois grupos, com vantagem para os estudantes que concluíram o curso em dois anos.

\section{Análise de Correlação entre os escores de integração acadêmica e o coeficiente de rendimento em disciplinas obrigatórias}

A seguir, apresentamos as correlações entre os escores de integração acadêmica no total e nas cinco dimensões por turma. Para analisar a relação entre essas variáveis, foi utilizado o coeficiente de correlação de Spearman, devido à ausência de distribuição Normal das variáveis. Para as correlações significativas grifadas em negrito na Tabela 5 e para a análise da força ou magnitude entre essas variáveis foi adotado o seguinte critério de classificação: 0 - não há correlação; 0,10 a 0,30 - correlação fraca; 0,40 a 0,60 - correlação moderada; 0,70 a 0,90 - correlação forte; e 1 correlação perfeita (Dancey \& Reidy, 2006).

Dentre os 18 pares de relação, em metade foram observados coeficientes de correlação significativos, sendo seis correlações de magnitude fraca e três moderadas. Em apenas um dos 
Tabela 4

Comparação dos escores de integração acadêmica e a situação acadêmica dos estudantes da turma de 2012

\begin{tabular}{|c|c|c|c|c|c|c|c|c|}
\hline Situação acadêmica & Variável & $\mathrm{n}$ & Média & D.P. & Mín. & Mediana & Máx. & Valor- $p$ \\
\hline \multirow[t]{12}{*}{ ATIVO } & AmbienteA & 17 & 4,18 & 0,38 & 3,63 & 4,25 & 4,75 & 0,646 \\
\hline & ComprCursA & 17 & 4,37 & 0,45 & 3,29 & 4,43 & 4,86 & 0,549 \\
\hline & HabEstudA & 17 & 3,09 & 0,46 & 2,1 & 3,3 & 3,8 & 0,051 \\
\hline & NaoObrigaA & 17 & 3,32 & 0,58 & 2,4 & 3,4 & 4,4 & 0,002 \\
\hline & CondiçõesA & 17 & 2,99 & 0,66 & 1,75 & 3,25 & 4 & 0,172 \\
\hline & EAVA_totalA & 17 & 3,63 & 0,29 & 2,97 & 3,65 & 3,94 & 0,299 \\
\hline & AmbienteB & 18 & 4,08 & 0,4 & 3,25 & 4,13 & 4,63 & 0,548 \\
\hline & ComprCursB & 18 & 4,21 & 0,52 & 2,86 & 4,29 & 4,86 & 0,310 \\
\hline & HabEstudB & 18 & 3,12 & 0,45 & 2,3 & 3,05 & 3,8 & 0,029 \\
\hline & NaoObrigaB & 18 & 3,06 & 0,52 & 1,6 & 3 & 3,8 & 0,121 \\
\hline & CondiçõesB & 18 & 2,76 & 0,62 & 2 & 2,63 & 4 & 0,504 \\
\hline & EAVA_totalB & 18 & 3,52 & 0,28 & 3,09 & 3,49 & 3,91 & 0,036 \\
\hline \multirow[t]{12}{*}{ CONCLUIU } & AmbienteA & 46 & 4,13 & 0,44 & 3,5 & 4 & 5 & \\
\hline & ComprCursA & 46 & 4,26 & 0,54 & 3,29 & 4,29 & 5 & \\
\hline & HabEstudA & 46 & 3,39 & 0,43 & 2,6 & 3,4 & 4,4 & \\
\hline & NaoObrigaA & 46 & 2,79 & 0,51 & 1,6 & 2,8 & 4,4 & \\
\hline & CondiçõesA & 46 & 2,7 & 0,66 & 1,25 & 2,75 & 3,75 & \\
\hline & EAVA_totalA & 46 & 3,57 & 0,3 & 3 & 3,53 & 4,26 & \\
\hline & AmbienteB & 36 & 4,21 & 0,47 & 3,25 & 4,13 & 5 & \\
\hline & ComprCursB & 36 & 4,37 & 0,51 & 3,14 & 4,36 & 5 & \\
\hline & HabEstudB & 36 & 3,51 & 0,59 & 2,4 & 3,45 & 4,9 & \\
\hline & NaoObrigaB & 36 & 3,39 & 0,71 & 2 & 3,2 & 5 & \\
\hline & CondiçõesB & 36 & 2,85 & 0,56 & 1,75 & 3 & 4 & \\
\hline & EAVA_totalB & 36 & 3,76 & 0,4 & 3,12 & 3,71 & 4,79 & \\
\hline
\end{tabular}

* Valor-P referente ao teste de Mann-Whitney para comparação das variáveis entre 2 grupos.

casos o sentido foi negativo, indicando que o envolvimento em atividades não obrigatórias estava, em grau fraco, inversamente relacionado com o desempenho acadêmico dos estudantes da turma 2012 ao final do primeiro ano de curso $(-0,38)$. As relações positivas, isto é, com o mesmo sentido de orientação entre as variáveis, mas em grau fraco, foram: habilidades do estudante da turma 2012 ao final do primeiro ano $(\rho=0,36)$; compromisso com o curso da turma de 2012 ao final do segundo ano $(\rho=0,31)$; ambiente universitário $(\rho=0,27)$, condições de estudo ( $\rho$ $=0,32)$ e integração acadêmica total $(\rho=, 32)$ da turma 2013 ao final do primeiro ano. Foram obtidas as seguintes correlações de magnitude moderada entre o desempenho acadêmico em disciplinas obrigatórias e medidas de integração: habilidades do estudante $(\rho=0,50)$ e Integração acadêmica total da turma de 2012 ao final do segundo ano; e habilidades do estudante da turma de $2013(\rho=0,52)$.

\section{Sintese dos resultados}

Em síntese, podemos dizer que os estudantes tinham, ao final do primeiro ano do curso, alta integração com o ambiente universitário e compromisso com o curso, e também média integração com a vida acadêmica. Por outro lado, tinham dificuldades quanto às condições para o estudo. 
Tabela 5

Correlações entre escores de integração e o coeficiente de rendimento em disciplinas obrigatórias por turma

\begin{tabular}{|c|c|c|c|c|}
\hline Turma & Variável & $\mathrm{n}$ & $\mathrm{CRO}^{*} \rho$ & $p$ \\
\hline \multirow[t]{12}{*}{ TURMA 2012} & AmbienteA & 65 & 0,01458 & 0,9083 \\
\hline & ComprCursA & 65 & 0,00593 & 0,9626 \\
\hline & HabEstudA & 65 & $\mathbf{0 , 3 6 2 1 7}$ & 0,003 \\
\hline & NaoObrigaA & 65 & $-0,38991$ & 0,0013 \\
\hline & CondiçõesA & 65 & 0,06826 & 0,589 \\
\hline & EAVA_totalA & 65 & 0,02281 & 0,8569 \\
\hline & AmbienteB & 57 & 0,24594 & 0,0652 \\
\hline & ComprCursB & 57 & 0,31713 & 0,0162 \\
\hline & HabEstudB & 57 & 0,50822 & $<0,0001$ \\
\hline & NaoObrigaB & 57 & 0,19762 & 0,1406 \\
\hline & CondiçõesB & 57 & 0,23801 & 0,0746 \\
\hline & EAVA_totalB & 57 & 0,48843 & 0,0001 \\
\hline \multirow[t]{6}{*}{ TURMA 2013} & AmbienteA & 63 & 0,27123 & 0,0315 \\
\hline & ComprCursA & 63 & 0,15713 & 0,2187 \\
\hline & HabEstudA & 63 & 0,52417 & $<0,0001$ \\
\hline & NaoObrigaA & 63 & $-0,15345$ & 0,2299 \\
\hline & CondiçõesA & 63 & 0,32133 & 0,0102 \\
\hline & EAVA_totalA & 63 & 0,32114 & 0,0103 \\
\hline
\end{tabular}

* $\rho=$ coeficiente de correlação de Spearman;

As turmas de 2012 e 2013 eram equivalentes quanto à sua integração total à vida universitária ao final do primeiro ano, mas diferenciavam-se nas dimensões ambiente universitário e compromisso com o curso (maior para a turma de 2012) e envolvimento em atividades não obrigatórias (maior para a turma de 2013).

Quanto à diferença de integração na turma de 2012 ao final do primeiro e segundo ano, observou-se aumento nas dimensões habilidades do estudante e envolvimento em atividades não obrigatórias e na integração total.

Ao analisar a situação atual da turma de 2012, notou-se que o maior escore na dimensão envolvimento em atividades não obrigatórias ao final do primeiro ano foi observado entre os estudantes ainda ativos no curso; e, na dimensão habilidades do estudante e no total, ao final do segundo ano, entre os estudantes concluintes no prazo de integralização esperado.

Foram também observadas correlações fracas e moderadas entre as medidas de integração e o coeficiente de rendimento em disciplinas obrigatórias dos estudantes das turmas de 2012 e 2013. Todas as dimensões se relacionaram, pelo menos em magnitude fraca em algumas das medidas, com o desempenho acadêmico. Destacase a correlação fraca negativa com o envolvimento em atividades não obrigatórias ao final do primeiro ano da turma de 2012 e as correlações de magnitude moderada entre o rendimento e a dimensão habilidades do estudante (segundo ano de 2012 e primeiro ano de 2013) e rendimento e integração total (segundo ano de 2012). 


\section{Discussão}

O presente estudo corrobora outras pesquisas que apontam a relevância da integração à vida universitária para o sucesso acadêmico e satisfação do estudante com sua formação (Almeida \& Soares, 2003; Carlotto, Teixeira, \& Dias, 2015; Cunha \& Carilho, 2005; Granado, Santos, Almeida, Soares, \& Guisande, 2005; Morgado, 2009; Pascarella \& Terenzini, 2005; Polydoro, 2000; Santos et al., 2010; Santos, Polydoro, Scortegagna, \& Linden, 2013; Scheleich, 2006; Vendramini et al., 2004, e outros). Os estudantes das turmas 2012 e 2013 tinham, ao final do primeiro ano do curso, escore médio de integração, tendo um aumento significativo até o final do segundo ano (turma 2012). Essa variável também diferenciou os grupos de estudantes ativos e concluintes, sendo maior entre os que concluíram o curso no tempo previsto de integralização, assim como, demonstrou correlação positiva com o desempenho acadêmico dos estudantes em disciplinas obrigatórias do curso.

A avaliação da integração do estudante que foi realizada nessa investigação também permite analisar os resultados em cinco diferentes dimensões do construto, oferecendo maior detalhamento sobre o processo. Os resultados obtidos permitem perceber a relevância da percepção do estudante sobre suas habilidades de aprendizagem e background resultante de escolaridade anterior para a formação atual. Além das dificuldades observadas em itens dessa dimensão, ao final do primeiro ano das duas turmas, ela correlacionou-se de forma positiva com o desempenho nas disciplinas obrigatórias para os estudantes das duas turmas e nas duas coletas realizadas. E ainda, os estudantes que concluíram o curso no prazo previsto diferenciaram-se significativamente dos ainda ativos por apresentarem média superior nessa dimensão. A trajetória anterior de formação do estudante e, mais especificamente, a percepção do estudante sobre sua experiência acadêmica no que se refere à base de conhecimentos e estratégias cognitivas para realizar o curso tem sido destacada em diferentes modelos teóricos sobre a vivência universitária, como variável de ingresso relevante para a integração e desenvolvimento do estudante no ensino superior (Pascarella \& Terenzini, 2005; Tinto, 1997). Porém, é preciso destacar que o perfil de ingresso dos estudantes pode ser alterado em decorrência de sua interação com o sistema acadêmico e social da instituição, e mais, deve ser objeto intencional de ação por parte da instituição e curso. Em particular, iniciativas que possam fortalecer a crença de autoeficácia acadêmica do estudante, isto é, a percepção de sua capacidade em organizar e executar cursos de ações requeridos para produzir realizações referentes aos aspectos intelectuais e de aprendizagem (Bandura, 1993), já que direciona o envolvimento, a persistência e o esforço, mesmo quando há obstáculos para a realização do objetivo proposto, afetando o desempenho (Bandura, 1993; Schunk, 1995). $\mathrm{Na}$ amostra estudada, houve a alteração das habilidades dos estudantes devido à imersão e esforço individual deles, do programa e dos professores que se engajaram na adaptação das disciplinas ao perfil dos alunos, em termos de conteúdos e dinâmicas pedagógicas.

A percepção do estudante em relação ao contexto físico, social, acadêmico e organizacional da instituição não se diferenciou quando coletada ao final do primeiro e segundo ano da turma de 2012, talvez porque já se apresentava alta na primeira coleta realizada. Na turma de 2013, observou-se média menor que a obtida ao final do primeiro ano da turma de 2012 e correlação positiva, mesmo que fraca, com o desempenho acadêmico dos estudantes. Vale ressaltar que, como refere Strange (1994), o papel do ambiente depende da percepção do estudante sobre ele. No presente estudo, por exemplo, observou-se diferença na percepção dos estudantes das turmas de 2012 e 2013 quanto ao ambiente universitário. Como o contexto universitário e suas interações são fontes potenciais de mudanças cognitivas e não cognitivas para os estudantes (Pascarella \& Terenzini, 2005), também devem ser objeto 
de atenção por parte dos gestores e professores. Nesse sentido, destacam-se os programas de acolhimento ao ingressante. No caso do curso em questão, por ter duração de dois anos, esse período de transição inicial requer uma postura ainda mais aberta e eficaz por parte da instituição e dos docentes para que se estabeleça diálogo com os estudantes sobre as oportunidades e desafios do curso, bem como, sobre as possibilidades de enfrentamento e integração.

Trajetória semelhante à observada com a dimensão ambiente universitário foi obtida quanto ao grau de certeza dos estudantes quanto à escolha de curso e de segurança quanto à formação, a saber: alta ao final do primeiro ano de 2012 com manutenção do escore alto na segunda coleta da turma de 2012 e menor quando coletada ao final do primeiro ano da turma de 2013. Também apresentou correlação positiva e fraca com o desempenho acadêmico em disciplinas obrigatórias - observado na segunda coleta da turma de 2012. Sabe-se que, além da relação com o investimento e desempenho, a variável compromisso com o curso tem papel significativo na decisão de permanência ou evasão do curso (Mercuri \& Polydoro, 2003; Tinto, 1975; 1997). Esta variável ganha ainda maior relevância quando se considera que no projeto do ProFIS, o ingresso na graduação está atrelado ao desempenho acadêmico obtido durante o curso de formação geral.

Ao analisar os itens da dimensão envolvimento dos estudantes em atividades não obrigatórias, isto é, sobre oportunidades de caráter acadêmico, científico, social, cultural ou esportivo propiciadas pela instituição, mas que não se caracterizam pelas exigências mínimas do curso, observouse que os estudantes relataram dificuldade de integração em relação às experiências não obrigatórias de natureza acadêmica ao final do primeiro ano do curso, o mesmo não sendo observado em atividades de outra natureza. A turma de 2013 termina seu primeiro ano de curso com maior média em envolvimento em atividades não obrigatórias que a turma de 2012 ao final do primeiro ano. Porém, essa média da turma de 2012 aumentou significativamente na segunda coleta, demonstrando maior integração às oportunidades não obrigatórias do ambiente universitário, provavelmente devido à transição inicial do curso e maior conhecimento dos eventos e recursos da instituição. Pesquisas como as de Kuh (1995), Capovilla e Santos (2001), e Fior e Mercuri (2003), dentre outras, demonstraram que a formação do estudante não se limita às atividades obrigatórias, sendo as atividades não obrigatórias muito relevantes para a formação do estudante, sendo associadas ao desenvolvimento do compromisso com o curso, desenvolvimento cognitivo e social e desempenho acadêmico. Os estudos de Mognon e Santos (2014); Teixeira, Castro, e Piccolo (2007) e Teixeira \& Gomes (2004) também relataram relação da realização de atividades não obrigatórias com o desenvolvimento de carreira e pessoal. Notou-se também que houve correlação negativa, mesmo que fraca, com o desempenho acadêmico e que na turma de 2012, os estudantes ativos tinham média significativamente maior nessa dimensão ao final do primeiro ano do que os estudantes que concluíram o curso. No estudo de Santos, Mognon, Lima, e Cunha (2011), também se observou relação negativa do envolvimento com atividades não obrigatórias e a motivação metas de realização para aprender. Sabe-se, também, que a realização de atividades não obrigatórias pode interferir no tempo de dedicação e no envolvimento do estudante nas atividades acadêmicas obrigatórias, pois pode gerar outra prioridade que não o próprio curso. Além disso, o impacto não é o mesmo para as diversas atividades disponíveis e nem para todos os estudantes da mesma forma (Almeida et al., 2000).

Por fim, a integração às condições para o estudo, que inclui suas condições físicas, disponibilidade de tempo e de investimento nas atividades acadêmicas, demonstrou ser a principal deficiência dos estudantes nas duas turmas do curso. E mais, mesmo baixa ao final do primeiro ano, não foi percebida melhora pelos estudantes ao final do segundo ano na turma de 2012; para os estudantes de 2013, se observou correlação 
positiva e fraca com o desempenho acadêmico nas disciplinas obrigatórias. Essa dimensão inclui aspectos muito relevantes para o processo de aprendizagem e desenvolvimento de autonomia dos estudantes frente aos estudos, especialmente por concentrar a as condições objetivas para o estudo e a competência dos estudantes para gestão de possíveis barreiras, como a distância e tempo de deslocamento entre moradia e a universidade, necessidade de conciliar atividades letivas (em classe e extraclasse) e atividades não letivas, disposição física para a concentração e o estudo (Vendramini et al., 2004). Para Astin (1993), em sua proposta sobre a teoria do envolvimento, o desenvolvimento e a aprendizagem do estudante dependem da quantidade e qualidade de energia física e psicológica despendida com as atividades acadêmicas e tempo de dedicação às mesmas. Sarriera, Paradiso, Schütz, e Howes (2012), em estudo sobre a integração ao contexto universitário de estudantes de diferentes instituições, reiteram a importância de oferecimento de apoio intencional pela instituição ao gerenciamento e organização do tempo de seus alunos, devido à relação dessa competência com a aprendizagem e o bem-estar físico e psicológico dos estudantes. Sabemos que dada à baixa consistência interna dessa dimensão, os dados precisam ser analisados com cautela, no entanto, reafirma-se a necessidade de atuação intencional por parte da instituição para além do oferecimento de bolsas de estudo. Entretanto, outros dados levantados pelo projeto de Avaliação Continuada do ProFIS apontam fatores que poderiam ser abordados pela coordenação do Programa. Os estudantes se ressentem de não terem um espaço exclusivo de estudo, dado que não possuem um prédio como os outros cursos da universidade. Isto poderia ser encaminhado pela reserva de espaços ou mesmo a divulgação dos espaços comuns de estudo da universidade para os alunos. Outro aspecto, mais difícil de ser equacionado devido aos limites orçamentários da universidade, refere-se ao oferecimento de moradia estudantil para evitar o longo deslocamento dos estudantes até o campus, dado que a maior parte mora com as famílias, muitas delas longe do campus e com poucas opções de transporte público. Para além dessas questões estruturais e ações docentes, iniciativas têm sido incrementadas a fim de fortalecer os processos autorregulatórios de estudo e aprendizagem dos estudantes, na parceria com o setor de Orientação Educacional do Serviço de Apoio ao Estudante da universidade e incentivo à frequência à disciplina eletiva Oficina de Autorregulação da Aprendizagem (Polydoro et al., 2015).

Os dados apontam que a integração do ensino superior compõe-se por fatores de natureza pessoal, contextual e a própria interação entre ambos. Diante do aumento do acesso ao ensino superior, como referem Upcraft, Gardner, \& Barefoot (2005), a instituição deve organizar a experiência acadêmica de forma a responder às necessidades e peculiaridades da nova população estudantil. Para os autores, é um desafio construir iniciativas que favoreçam os tipos de ambientes necessários para o sucesso de todos os estudantes. Mas, anunciam que a base para isso está em ambientes de aprendizagem que promovam desafio e apoio aos alunos, evitando desequilíbrio entre os dois. Ou, como refere Nico (2000), as instituições de ensino superior devem promover condições apropriadas para que seus estudantes vivenciem uma adequada e satisfatória sensação de conforto acadêmico, isto é, uma percepção de equilíbrio pessoalmente adequado nas diferentes dimensões do contexto em que se encontra.

Destacam-se como limitações do presente estudo: a) delimitações do instrumento de coleta de dados que reflete a percepção do estudante conforme um determinado conjunto de experiências universitárias; b) somente duas medidas longitudinais e em apenas uma das turmas; c) redução do desempenho acadêmico do estudante no curso no coeficiente de rendimento em disciplinas obrigatórias. Como continuidade desse estudo sugere-se a avaliação do efeito condicional das características dos estudantes no impacto da experiência de formação e o acompanhamento dos estudantes até sua conclusão do Programa, ingresso e trajetória de graduação. 


\section{Referências}

Almeida, L. S., \& Soares, A. P. (2003). Os estudantes universitários: sucesso escolar e desenvolvimento psicossocial. In E. Mercuri, \& S.A.J. Polydoro (Orgs.), Estudante universitário: características e experiências de formação (pp. 15-40). Taubaté: Cabral Editora e Livraria Universitária.

Almeida, L. S., Soares, A. P., Vasconcelos, R., Capela, J. V., Vasconcelos, J. B., Corais, J. M., \& Fernandes, Á. (2000). Envolvimento extracurricular e ajustamento acadêmico: um estudo sobre as vivências dos estudantes universitários com e sem funções associativas. In A.P. Soares, A. Osório, J.V. Capela, L.S. Almeida, R.M. Vasconcelos, \& S.M. Caires (Eds.), Transição para o ensino superior (pp. 167-188). Braga: Universidade do Minho, Conselho Universitário.

Almeida, L. S., Gonçalves, A., Soares, A. P., Marques, A. P., Fernandes, E., Machado, C., ... \& Vasconcelos, R. (2004). Transição, adaptação e rendimento acadêmico de jovens no Ensino Superior (Relatório Final de Projeto). Braga: Universidade do Minho.

Andrade, C. Y., Gomes, F. A. M., Knobel, M., \& Carneiro, A.M. (2012). Programa de Formação Interdisciplinar Superior: um novo caminho para a educação superior. Revista Brasileira de Estudos Pedagógicos, 93(235), 698719. Recuperado de http://www.rbep.inep.gov.br/index. $\mathrm{php} / \mathrm{rbep} / \mathrm{article} / \mathrm{view} / 412 / 400$

Andrade, C. Y., Gomes, F. A. M., Pedrosa, R. H. L., Silva, A. M. A. C., Velloso, L. A., Pereira, E. A., \& Celani, G. (2013). ProFIS: A new paradigm for higher education in Brazil. Journal of Widening Participation and Lifelong Learning, 15(3), 22-46. doi: 10.5456/WPLL.15.3.22

Astin, A. W. (1993). What matters in college? Four critical years revisited. San Francisco: Jossey-Bass.

Bandura, A. (1993). Perceived self-efficacy in cognitive development and functiong. Educational Psychologist, 28(2), 117-148. doi: 10.1207/s15326985ep2802_3

Capovilla, S. L., \& Santos, A. A. A. (2001). Avaliação da influência de atividades extramuros no desenvolvimento pessoal de universitários. Psico-USF, 6(2), 49-58. Recuperado de http://bases.bireme.br/cgi-bin/wxislind. exe/iah/online/

Carlotto, R. C., Teixeira, M. A. P., \& Dias, A. C. G. (2015). Adaptação acadêmica e coping em estudantes universitários brasileiros. Psico-USF, 20(3), 421-432. doi: 10.1590/1413-82712015200305

Carneiro, A. M., Andrade, C. Y., \& Gonçalves, M. L. (2012). Formação interdisciplinar e inclusão social - o primeiro ano do ProFIS. Ensino Superior Unicamp, 3, 24-38.

Carneiro, A. M. Andrade, C. Y., \& Telles S. M. B. S. (2012). Avaliação continuada do programa de formação interdisciplinar superior da Unicamp: proposta metodológica. Revista Brasileira de Monitoramento e Avaliação, 1, 26-45.
Carneiro, A. M. Camelo, A. P., Andrade, C. Y., \& Telles S. M. B. S. (2015). Interdisciplinary Higher Education Program (ProFIS): Challenges and Opportunities. In R. T. Teranishi, L. B. Pazich, M. Knobel \& W. R. Allen (Eds.) Advances in education in diverse communities: research, policy and praxis. $1^{\mathrm{a}} \mathrm{ed}$. (pp. 265-282). Bingley: Emerald Group Publishing Limited.

Cunha, S. M., \& Carrilho, D. M. (2005). O processo de adaptação ao ensino superior e o rendimento acadêmico. Psicologia Escolar e Educacional, 9(2), 215-224. Recuperado de http://pepsic.bvsalud.org/scielo.php?script=sci_art text\&pid=S1413-85572005000200004

Dancey, C. P., \& Reidy, J. (2006). Estatística sem matemática para psicologia. Porto Alegre: Artmed.

Dey, E. L., \& Hurtado, S. (1995). College impact, student impact: a reconsideration of the hole of students within American higher education. Higher Education, 30(2), 207-223. doi: 10.1007/BF01384097

Fior, C., \& Mercuri, E. (2003). Formação universitária: o impacto das atividades não obrigatórias. In E. Mercuri, \& S. A. J. Polydoro (Orgs.), Estudante universitário: características e experiências de formação (pp. 129-154). Taubaté, SP: Cabral Editora e Livraria Universitária.

Granado, J. I. F., Santos, A. A. A., Almeida, L. S., Soares, A. P., \& Guisande, M. A. (2005). Integração académica de estudantes universitários: contributos para a adaptação e validação do QVA-r no Brasil. Psicologia e Educação, 4(2), 31-42. http://repositorium.sdum.uminho.pt/ handle/1822/12089

Kuh, G. D. (1995). The other curriculum: out-of-class experiences associated with student learning and personal development. Journal of Higher Education, 66(2), 23-55. Recuperado de http://www.jstor.org/ stable/2943909?seq=1\#page_scan_tab_contents

Mercuri, E., \& Polydoro, S. A. J. (Orgs.). (2003). Estudante universitário: características e experiências de formação. Taubaté, SP: Cabral Editora e Livraria Universitária.

Mognon, J. F., \& Santos, A. A. A. (2014). Vida acadêmica e exploração vocacional em universitários formandos: relações e diferenças. Estudos e Pesquisas em Psicologia, 14, 89-106.

Morgado, N. F. (2009). Retenção de alunos e persistência em instituições de ensino superior: uma revisão da literatura. Paradigma, XXX, 39-61.

Nico, J. B. (2000). O conforto académico do (a) caloiro (a). In A. P. Soares, A. Osório, J. V. Capela, L.S. Almeida, R. M. Vasconcelos, \& S. M. Caires (Eds.), Transição para o ensino superior (pp. 161-166). Braga: Universidade do Minho, Conselho Universitário.

Pascarella, E. T., \& Terenzini, P. T. (2005). How college affects students: a third decade of research. $2^{\mathrm{a}}$ ed. San Francisco: Jossey-Bass. 
Polydoro, S. A. J. (2000). O trancamento de matrícula na trajetória acadêmica do universitário: condições de saída e de retorno à instituição. (Tese de Doutorado). Universidade Estadual de Campinas, Campinas, Brasil.

Polydoro, S. A. J., Pelissoni, A. M. S., Coralina, M. C., Emilio, E. R. V., Dantas, M. A., \& Rosario, P. (2015). Promoção da autorregulação da aprendizagem na universidade: percepção do impacto de uma disciplina eletiva. Revista Educação PUC-Campinas, 20(3), 201-213. http://periodicos.puc-campinas.edu.br/seer/index.php/ reveducacao/article/view/2877/2216

Pró-Reitoria de Graduação. (2010). Projeto pedagógico: programa de formação interdisciplinar superior. Campinas: Universidade Estadual de Campinas.

Santos, A. A. A., Mognon, J. F., Lima, T. H., \& Cunha, N. B. (2011). A relação entre vida acadêmica e a motivação para aprender em universitários. Psicologia Escolar e Educacional, 15(2), 283-290. http://www.scielo.br/pdf/ pee/v15n2/v15n2a10

Santos, A. A. A., Polydoro, S. A. J., Scortegagna, S. A., \& Linden, M. S. S. (2013). Integração ao ensino superior e satisfação acadêmica universitários. Psicologia: Ciência e Profissão, 33(4), 780-793. doi: 10.1590/S141498932013000400002

Santos, A. A. A., Polydoro, S. A. J., Teixeira, M. A. P., \& Bardagi, M. P. (2010). Avaliação da integração do aluno ao ensino superior no contexto brasileiro. In A. A. A. Santos, F. F. Sisto, E. Boruchovitch, \& E. Nascimento (Eds.), Perspectivas em avaliação psicológica (pp. 165-188). São Paulo: Casa do Psicólogo.

Sarriera, J. C., Paradiso, A. C., Schütz, F. F., \& Howes, G. P. (2012). Estudo comparativo da integração ao contexto universitário entre estudantes de diferentes instituições. Revista Brasileira de Orientação Profissional, 13(2), 163-172. http://pepsic.bvsalud.org/ pdf/rbop/v13n2/04.pdf

Schleich, A. L. R. (2006). Integração na educação superior e satisfação acadêmica de estudantes ingressantes e concluintes. (Dissertação de Mestrado). Universidade
Estadual de Campinas, Campinas, Brasil.

Schunk, D. H. (1995). Self-efficacy and education and instruction. In J.E. Maddux (Ed.), Self-efficacy, adaptation and adjustment- theory, research and application (pp. 281303). New York: Plenum Press.

Strange, C. (1994). Student development: the evolution and status of an essential idea. Journal of College Student Development, 35(6), 399-412. Recuperado de http:// psycnet.apa.org/psycinfo/1995-27175-001

Teixeira, M. A. P., Castro, G. D., \& Piccolo, L. R. (2007). Adaptação à universidade em estudantes universitários: um estudo correlacional. Integração em Psicologia, 11(2), 211-220. Recuperado de http://jefersonmorieljunior. pbworks.com/w/file/fetch/51896088/artigo\%20 02 _pesquisa $\% 20$ com $\% 20$ descricao\% 20 e $\% 20$ correlacao_7466-37513-1-PB.pdf

Teixeira, M. A. P., \& Gomes, W. B. (2005). Decisão de carreira entre estudantes em fim de curso universitário. Psicologia: Teoria e Pesquisa, 21, 327-334.

Tinto, V. (1975). Dropout from higher education: A theoretical synthesis of recent research. Review of educational research, 45(1), 89-125. Recuperado de http://www.jstor.org/stable/1170024?seq=1\#page_ scan_tab_contents

Tinto, V. (1997). Classrooms as communities. Exploring the educational character of student persistence. Journal of Higher Education, 68(6), 599-623. doi: 10.2307/2959965

Upcraft, M. L., Gardner, J. N., \& Barefoot, B. O. (2005). Introduction: the first year of college revisited. In M. L. Upcraft, J. N. Gardner, \& B.O. Barefoot (Orgs.), Challenging and supporting the first-year student: A handbook for improving the first year of college (pp. 1-12). San Francisco: Jossey-Bass.

Vendramini, C. M. M., Santos, A. A. A., Polydoro, S. A. J., Sbardelini, E. T. B, Serpa, M. N. F., \& Natário, E. G. (2004). Construção e validação de uma escala sobre avaliação da vida acadêmica (EAVA). Estudos de Psicologia (Natal), 9(2), 259-268. Recuperado de http://www.scielo. br/pdf/epsic/v9n2/a07v9n2.pdf 